\title{
Registration of 2D to 3D Joint Images Using Phase-Based Mutual Information
}

\author{
Rupin Dalvi $^{\mathrm{a}}{ }$, Rafeef Abugharbieh ${ }^{\mathrm{a}}$, Mark Pickering ${ }^{\mathrm{b}}$, Jennie Scarvell ${ }^{\mathrm{c}}$, Paul Smith ${ }^{\mathrm{d}}$ \\ ${ }^{a}$ Biomedical Signal and Image Computing Lab, University of British Columbia, Canada \\ ${ }^{\mathrm{b}}$ School of Info. Tech. and Elect. Eng., Australian Defence Force Academy, Australia

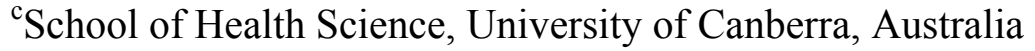 \\ ${ }^{\mathrm{d}}$ Trauma and Orthopaedic Research Unit, The Canberra Hospital, Australia
}

\begin{abstract}
Registration of two dimensional to three dimensional orthopaedic medical image data has important applications particularly in the area of image guided surgery and sports medicine. Fluoroscopy to computer tomography (CT) registration is an important case, wherein digitally reconstructed radiographs derived from the CT data are registered to the fluoroscopy data. Traditional registration metrics such as intensity-based mutual information (MI) typically work well but often suffer from gross misregistration errors when the image to be registered contains a partial view of the anatomy visible in the target image. Phase-based MI provides a robust alternative similarity measure which, in addition to possessing the general robustness and noise immunity that MI provides, also employs local phase information in the registration process which makes it less susceptible to the aforementioned errors. In this paper, we propose using the complex wavelet transform for computing image phase information and incorporating that into a phase-based MI measure for image registration. Tests on a CT volume and 6 fluoroscopy images of the knee are presented. The femur and the tibia in the CT volume were individually registered to the fluoroscopy images using intensity-based MI, gradient-based MI and phase-based MI. Errors in the coordinates of fiducials present in the bone structures were used to assess the accuracy of the different registration schemes. Quantitative results demonstrate that the performance of intensity-based MI was the worst. Gradient-based MI performed slightly better, while phase-based MI results were the best consistently producing the lowest errors.
\end{abstract}

KEYWORDS: Registration, multiresolution and wavelets, other (2D-3D registration).

\section{INTRODUCTION}

Registration of two dimensional (2D) to three dimensional (3D) bone and joint image data is gaining importance in many fields including (image guided) orthopaedic surgery and analysis and sports medicine. Real time data acquired during surgery or physical examination is mostly 2D (usually fluoroscopic). This 2D data typically needs to be registered to corresponding previously scanned 3D computer tomography (CT) data in order to facilitate the understanding of joint and bone motion in 3D. In many cases the registration, being that of the joints, is piecewise rigid and involves registration of a moving image that is a subset of the target image. For instance, while assessing movement of the knee, the femur and the tibia obtained from the CT scan may be registered separately to the whole knee image obtained from the fluoroscopy data.

The typical approach involved in rigid registration of 2D fluoroscopy images to 3D CT images involve the generation of a simulated x-ray image from the CT scan by summing the voxel intensities of the CT volume along a certain direction. This simulated 2D image is known as a digitally reconstructed radiograph or DRR. This image is then compared to the fluoroscopy images with the help of a similarity metric that indicates how closely the two images are aligned. This procedure is performed iteratively, rotating and translating the CT volume for each DRR construction until the similarity metric is optimized. While the image comparison (matching) may be made on the basis of key features or

1 Email: rupind@ece.ubc.ca . URL: http://bisicl.ece.ubc.ca/. Tel: + 1604 822-8851. Fax: +1 604 822-5949

Medical Imaging 2007: Image Processing, edited by Josien P. W. Pluim, Joseph M. Reinhardt, Proc. of SPIE Vol. 6512, 651209, (2007) · 1605-7422/07/\$18 · doi: 10.1117/12.709118 
surfaces acquired from the images as in [1], these methods tend to be difficult to automate, and the acquisition of the features is usually difficult and not very robust. On the other hand, registration performed using similarity metrics calculated on the entire data (i.e. using all pixels of the fluoroscopy and DRR images) such as in [2]-[6] is easier to automate and is much more robust. However, the selection of a proper similarity metric is important. An overview of the metrics used in 2D to 3D registration was made by Penney et al [7], wherein they investigated the following metrics on phantom data: normalized cross correlation, entropy of the difference image, intensity based mutual information (MI), gradient correlation, pattern intensity and gradient difference. Their conclusion was that (intensity) MI, which was simultaneously proposed by [9] and [10], was not well suited for 2D to 3D registration problems. However, Russakoff et al [8], investigated the same metrics on clinical spine data and reported good registration using intensity MI. Russakoff attributed the improvement in the performance of the intensity MI to the fact that the images used in their study were of higher resolution and hypothesized that different metrics perform differently on different data.

One thing to be noted in all the above mentioned approaches is that they register images to volumes that depict the same field of view of the anatomy. In other words, if, for instance, the fluoroscopic image is of the femur, then the CT image is also of the femur alone e.g. [3]. However, many registration problems involve registration of a target image (e.g. femur in CT data) that is a subset of the reference image (e.g. the whole knee joint in fluoroscopic data). In such a scenario, commonly used metrics such as cross correlation or intensity-based MI are prone to suffer from gross misregistration errors. These errors may be dealt with by basing the similarity metric on image attributes other than intensity. Using local phase information of the images is an option initially suggested by [13]. This has been used in [14] for monomodal volume registration. Mellor and Brady [11] have used the monogenic signal to generate local phase images from which they have calculated mutual information (i.e. phase based MI or PMI) and used that as their similarity metric in the registration procedure.

This paper deals with the rigid 2D to 3D registration of joint images (CT to fluoroscopy) by registering the femur and the tibia in the CT volume separately to the full fluoroscopic image. In order to overcome the problems described earlier, our approach is to generate phase images of the DRRs and fluoroscopic images which are then used to generate a PMI similarity metric for registration. We use the dual tree complex wavelet transform introduced by Kingsbury [12] for the calculation of local phase for its efficiency and good directional selectivity.

The rest of this paper is organized as follows: Section 2.1 briefly describes the complex wavelet transform and its use in generating image phase information. Section 2.2 presents details of our imaging and data acquisition and the registration procedure. Section 3 discusses the results on our CT and fluoroscopic joint data and compares those to results of registration approaches that employ other metrics.

\section{METHODS}

\subsection{Phase-Based Similarity Metric}

We propose the use of local phase information for the registration of 2D fluoroscopic data to 3D CT volumes. To generate the phase images, we use the dual-tree complex wavelet transform (DTCWT) introduced by Kingsbury [12], which is an efficient way to calculate phase images at various scales. The DTCWT also has 6 directions of selectivity at each level, making the transform highly sensitive to diagonal features. This transform employs Q (quadrature) shift complex wavelets (CWs). These DTCWTs are implemented using two discrete wavelet transforms (DWTs) on the same data. The original image data is decomposed using one wavelet filter along one 'branch' of the 'tree' and another wavelet filter along another branch, hence the name dual tree. These filters are designed in such a manner that the wavelet used in one branch is approximately the Hilbert Transform of the wavelet in the other branch. The coefficients in one branch can then be considered the real part of a complex wavelet transform with the coefficients in the other branch considered as the imaginary part. The basis functions of this transform have 6 distinct directions which provide the aforementioned 6 directions of selectivity at each level (Figure 1). 


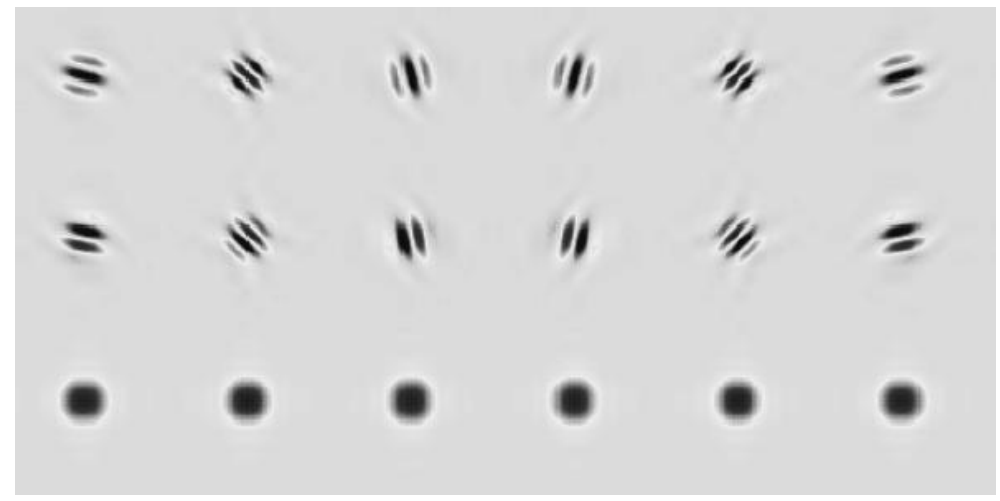

Figure 1: Real (top), imaginary (middle), and magnitude (bottom) parts of basis functions of two-dimensional impulse responses for the six bandpass bands of the 2D Q shift complex wavelets. The CWT has 6 filters, all of which are directionally selective $\left(-15^{\circ},-45^{\circ},-75^{\circ},+15^{\circ},+45^{\circ},+75^{\circ}\right)$, whereas the real wavelets have 3 filters, only 2 of which are directionally selective.

In this paper we use the complex wavelet transform to compute image phase information and incorporate it into a PMI measure for registering medical image data of the joints. Given a DRR of the CT volume (image A) and a fluoroscopic image (image B), we apply the DTCWT to both and analyze them to 3 levels. (Both images are, for this analysis, taken to be of the same size.) Since the transform has 6 directionally selective filters, this gives 6 complex valued wavelet coefficient images each of size $m \times n$. The 6 wavelet domain images representing image A and the 6 representing B are then concatenated (separately for A and B) to form two images of size $2 m \times 3 n$ each. The arrangement of the individual images in the concatenated images is such that the wavelet image for a particular filter for $\mathrm{A}$ is in the same relative position in the concatenated image as the corresponding filter image for $\mathrm{B}$ in its own concatenated image. Each complex wavelet coefficient for image A can be expressed as:

$$
M_{A}(i, j) e^{j \theta_{A}(i, j)}
$$

where $(i, j)$ indicates the position of the coefficient in the concatenated coefficient image (at level 3 in this work), $M_{A}$ indicates the magnitude at $(i, j)$, and $\theta_{A}$ indicates the phase. Similarly each complex wavelet coefficient for image B can be written as:

$$
M_{B}(i, j) e^{j \theta_{B}(i, j)}
$$

A joint histogram can now be generated using the following equation for each coefficient position $(i, j)$ :

$$
p\left(\theta_{A}(i, j), \theta_{B}(i, j)\right)=p\left(\theta_{A}(i, j), \theta_{B}(i, j)\right)+1
$$

where $p(a, b)$ is the joint probability of $a$ and $b$. A phase mutual information similarity metric can then be calculated using the following formula.

$$
P M I=\sum_{u=1}^{N} \sum_{v=1}^{N} p(u, v) \log \left(\frac{p(u, v)}{\left(\sum_{u=1}^{N} p(u, v) \sum_{v=1}^{N} p(u, v)\right)}\right)
$$

where $N$ is the number of bins used to generate the joint histogram. 


\subsection{Registration Procedure}

CT and fluoroscopy data of a cadaverous knee with 14 fiducials in the form of tantalum beads on both bones (femur and tibia with 7 fiducials each) was acquired. Tantalum beads show up clearly in both imaging modalities. It is important to note that the beads were not used to aid the registration process rather only used to verify the accuracy of the registration algorithm. Registration errors were measured as the distance between corresponding fiducials in the DRRs of the registered CT volume and the corresponding fluoroscopic images. For the knee used in our study, one CT volume and 6 fluoroscopy images were tested in the registration experiments. The femur and tibia bone areas were individually segmented from the CT data and then registered to the fluoroscopy images using the procedure outlined below. Three different metrics where contrasted for comparison purposes: IMI (intensity-based mutual information), GMI (gradientbased mutual information), and our proposed PMI (phase-based mutual information).

In order to reduce computational cost, a coarse manual prealignment (guided by the tantalum beads) was done to bring the images into a rough initial alignment. This facilitated setting the search area for the PMI registration to a relatively small local region (of $\pm 3^{\circ}$ and \pm 3 pixels). The CT volume (either the femur or the tibia) was then rotated and translated (by \pm 3 degrees and \pm 3 pixels respectively) around and along all three directions. Then this transformed volume was collapsed along the $\mathrm{z}$ direction in order to generate the DRR. (Actually, the translations were performed after the DRR acquisition in order to save time.) Then the PMI of the images was calculated as stated above. At the end of all the combinations of rotations and translations, the combination for which the PMI was highest was taken as the correct transform.

\section{RESULTS}

The performance of the proposed PMI metric was contrasted to IMI and GMI similarity metrics, where mutual information of the intensity and gradient images of the fluoroscopy and DRR images, respectively, was used instead of our proposed complex wavelet derived phase based metric. Registration accuracy was assessed using the bead (fiducial) locations in the registered image pairs.

Results show that the performance of IMI was the worst among the three registration metrics. GMI performed relatively better while our proposed complex wavelet-based PMI similarity measure performed best consistently giving lower registration errors. Graphs showing the registration errors obtained with the three tested metrics on our data (CT volume registered to each fluoroscopic image) are shown in Figures 3 and 4. A summary of the registration results is shown in Table. 1. Figures 5-7 show examples of registration results. Figure 5 shows the CT volume, a sample fluoroscopic image and DRRs of the femur and the tibia generated after segmenting the two bones in the CT data. Figure 6 shows a checkerboard pattern of sample femur images (DRR and fluoroscopic) in their unregistered state as well as after registration using IMI, GMI and PMI respectively. Figure 7 shows the corresponding images for the tibia.

\begin{tabular}{|l|c|c|c|c|}
\hline Registration Method & $\begin{array}{c}\text { Mean horizontal } \\
\text { pixel error: } \text { tibia }\end{array}$ & $\begin{array}{c}\text { Mean vertical } \\
\text { pixel error: } \text { tibia }\end{array}$ & $\begin{array}{c}\text { Mean horizontal } \\
\text { pixel error: } \text { femur }\end{array}$ & $\begin{array}{c}\text { Mean vertical pixel } \\
\text { error: } \text { femur }\end{array}$ \\
\hline None & 1.23 & 0.83 & 1.3 & 0.69 \\
\hline IMI & 1.83 & 1.60 & 0.93 & 2.90 \\
\hline GMI & 1.00 & 0.70 & 0.76 & 0.62 \\
\hline PMI (proposed) & $\mathbf{0 . 1 7}$ & $\mathbf{0 . 2 7}$ & $\mathbf{0 . 4 3}$ & $\mathbf{0 . 3 8}$ \\
\hline
\end{tabular}

Table 1: Mean error in all fiducial markers (across all images) for various similarity metrics after registration of fluoroscopic images to CT based DRRs. IMI: intensity-based mutual information, GMI: gradient-based mutual information, PMI: our proposed phase-based mutual information. Lowest errors indicated in bold face. 


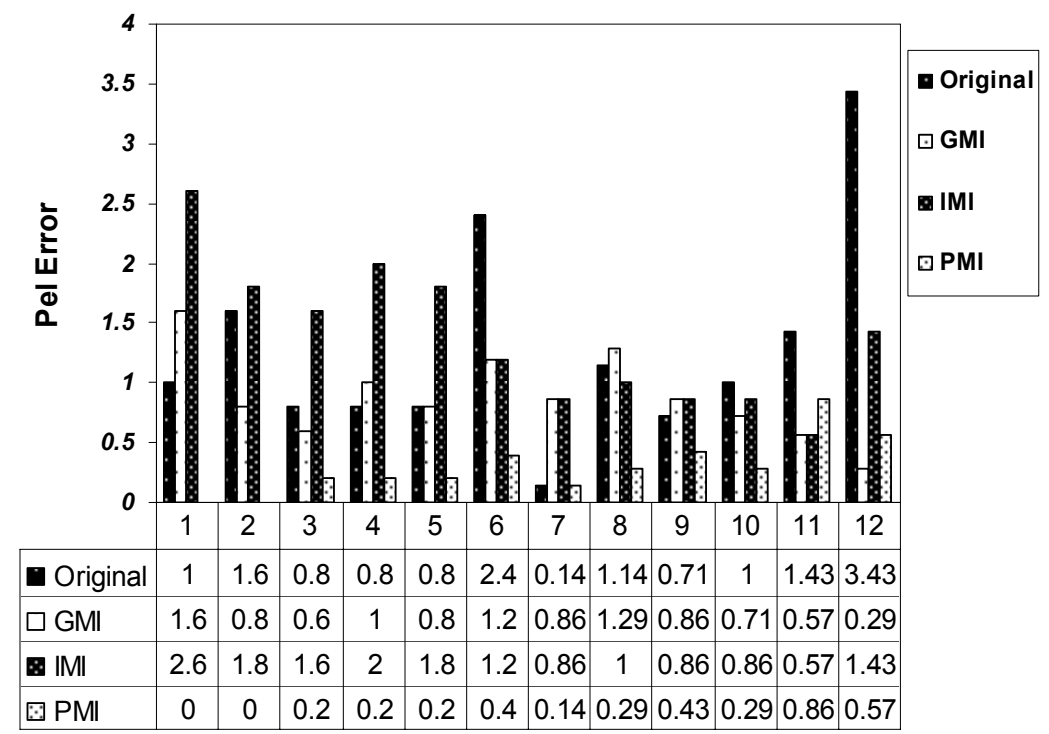

Fluoro. Image Number (Tibia:1-6; Femur:7-12)

Figure 3: Horizontal registration error for various metrics when registering CT data of the tibia (columns 1-6) and femur (columns 7-12) to 6 fluoroscopy images.

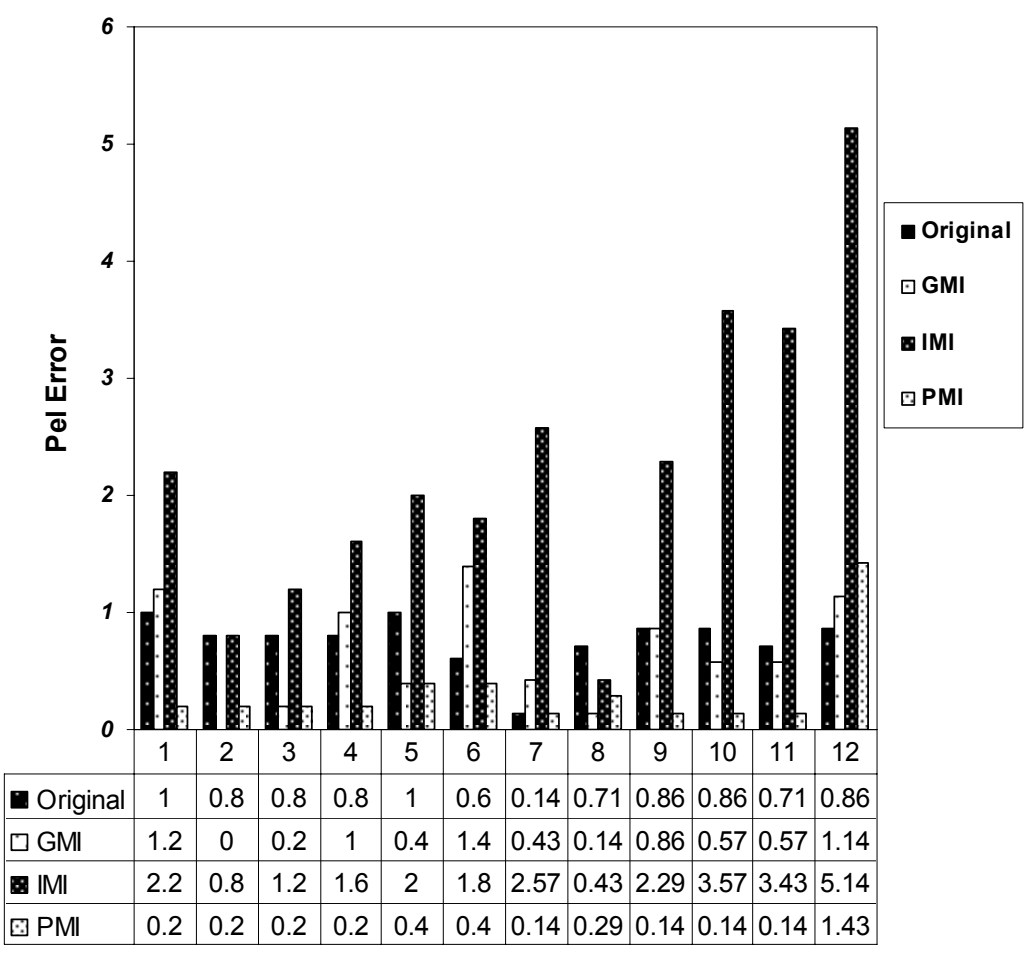

Fluro. Image Number (Tibia:1-6; Femur:7-12)

Figure 4: Vertical registration error for various metrics when registering CT data of the tibia (columns 1-6) and femur (columns 7-12) to 6 fluoroscopy images. 


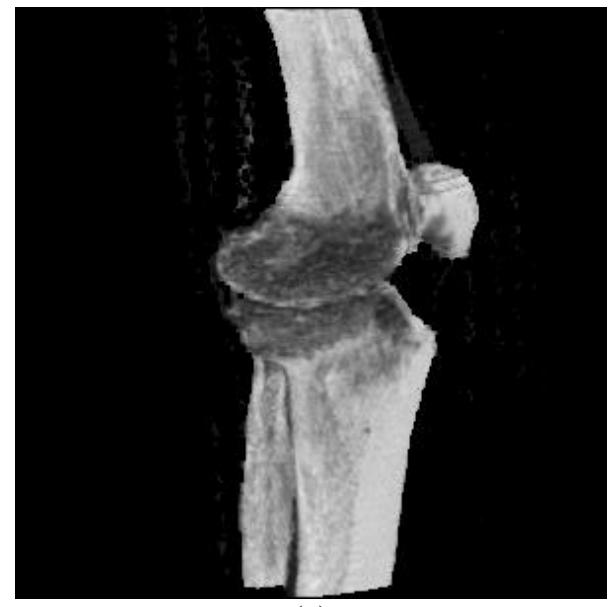

(a)

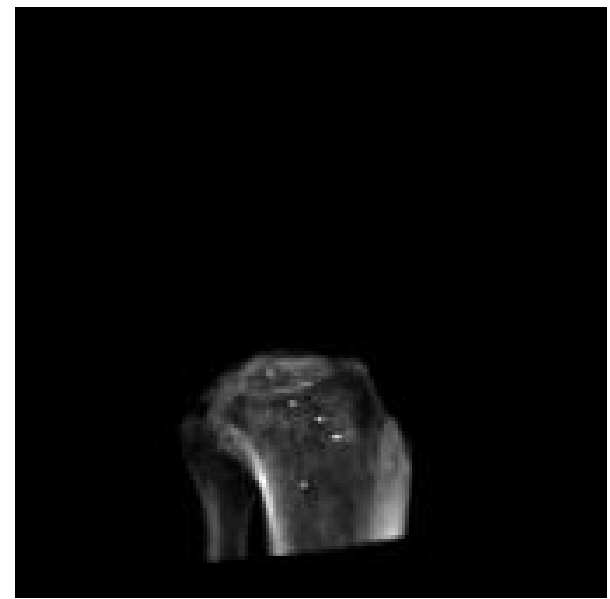

(c)

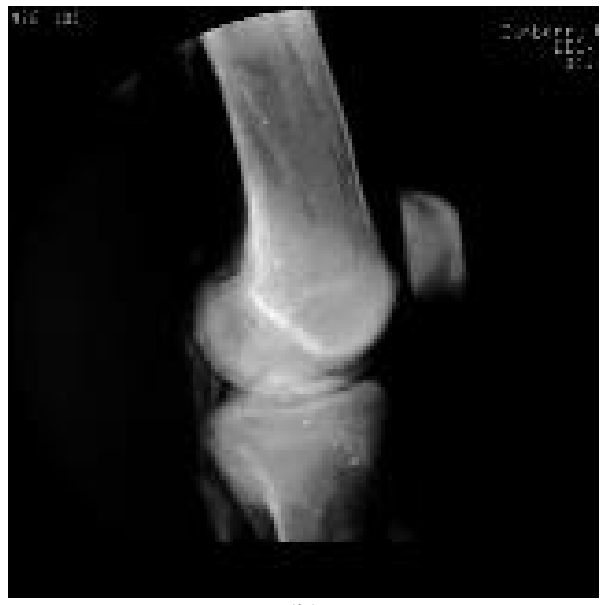

(b)

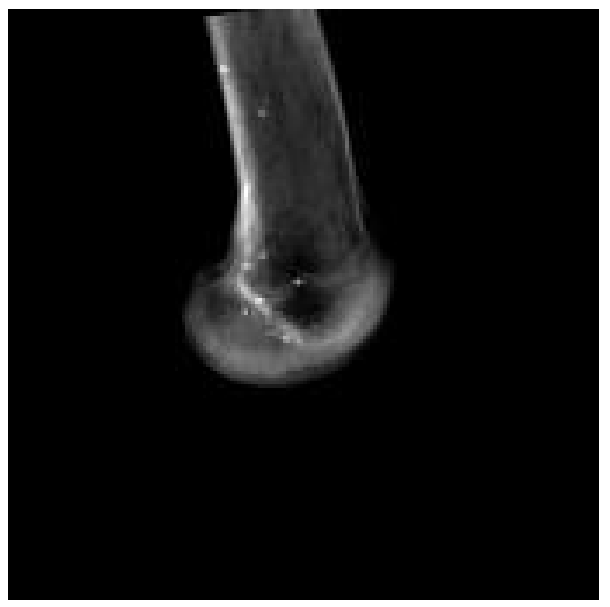

(d)

Figure 5: (a) 3D rendering of knee joint from CT data (thresholded to show only bone). (b) Example fluoroscopic image. (c) DRR of the tibia made from the CT data. (d) DRR of the femur. 


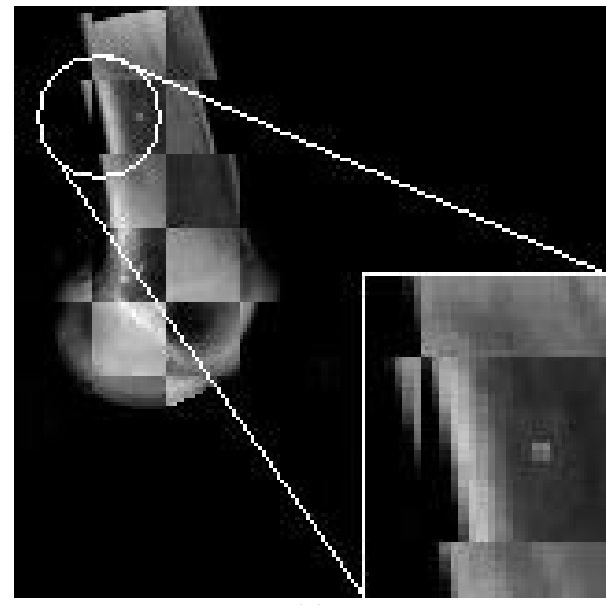

(a)

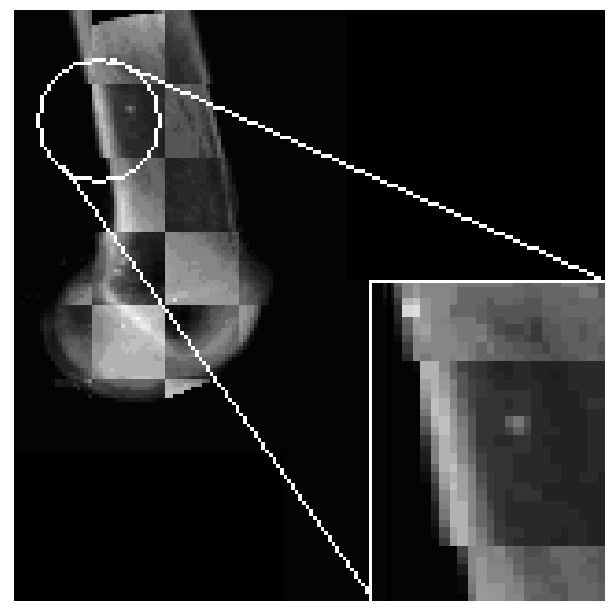

(c)

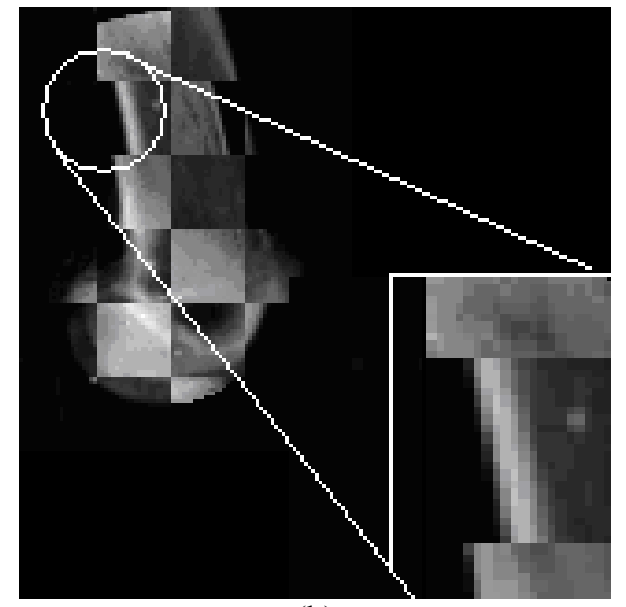

(b)

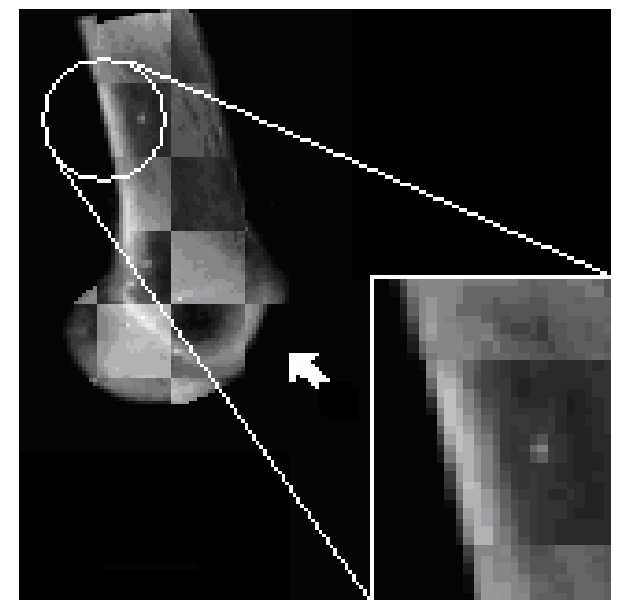

(d)

Figure 6: (a) Checkerboard of unregistered femur images (DRR + Fluoro Image 4). (b) Corresponding registration result of femur images done using IMI. (c) Registration of femur images done using GMI. (d) Registration of femur images done using our proposed PMI metric. (The area pointed at looks misregistered because there is a certain region of the bone (or cartilage) in that area that is seen in the DRR but is invisible in the fluoroscopic image.) 


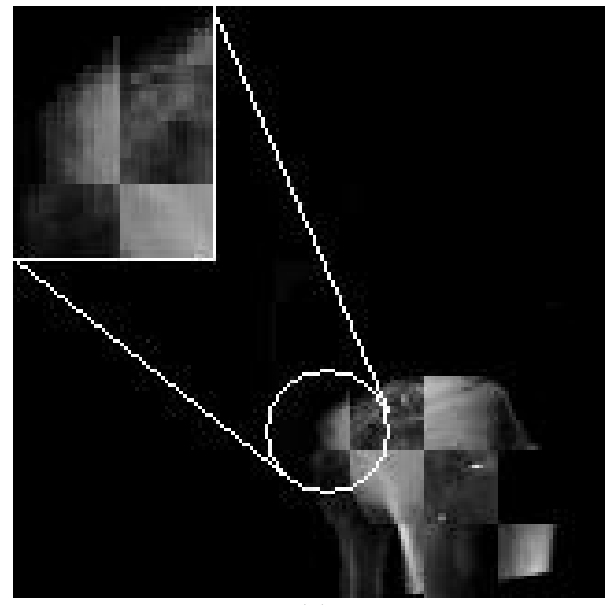

(a)

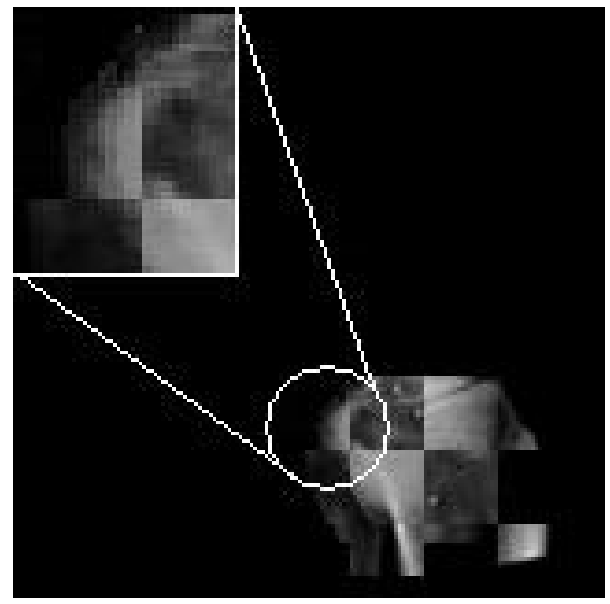

(c)

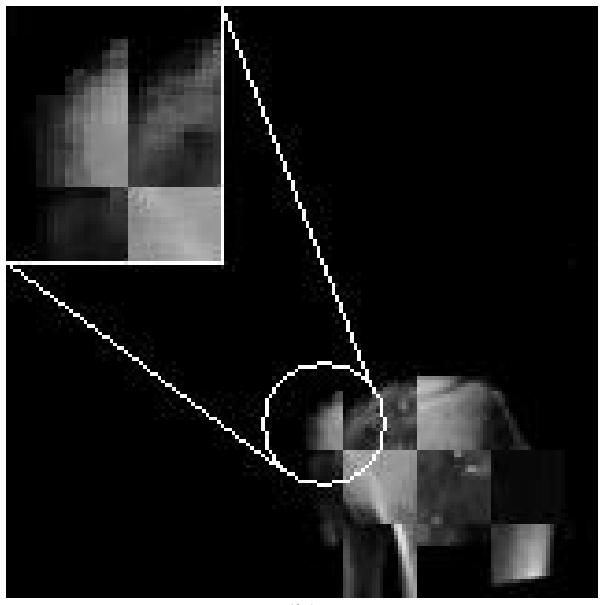

(b)

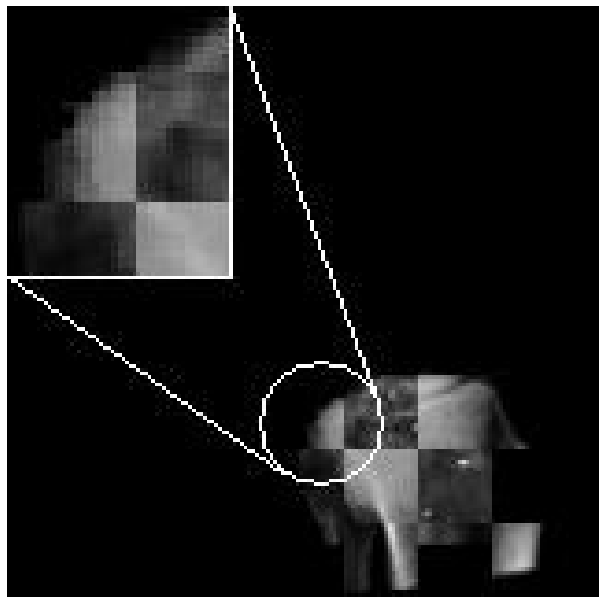

(d)

Figure 7: (a) Checkerboard of unregistered tibia images (DRR+Fluor. Image 3). (b) Corresponding registration result of tibia images done using IMI. (c) Registration of tibia images using GMI. (d) Registration of tibia images using our proposed PMI metric.

\section{CONCLUSIONS}

This paper proposed the use of phase-based mutual information derived from complex wavelets (DTCWT) as a robust similarity metric for $2 \mathrm{D}$ to $3 \mathrm{D}$ image registration of joint images. The proposed metric was tested on bone and joint images in a fluoroscopy to CT registration framework. Quantitative results indicate superior performance of the proposed metric over conventional metrics such as intensity-based and gradient-based mutual information. The presented metric has the general robustness and noise immunity that MI provides. However, due to the nature of the DTCWT, it also has local phase information that makes it less susceptible to misregistration encountered when the image to be registered depicts a subset of the target anatomy.

\section{REFERENCES}

[1] Maurer CR Jr, Maciunas RJ, Fitzpatrick JM, “ Registration of head CT images to physical space using a weighted combination of points and surfaces," IEEE Trans Med Imaging, 17(5):753-61, 1998..

[2] L. Lemieux, R. Jagoe, D. R. Fish, N. D. Kitchen, and D. G. T. Thomas, “A patient-to-computed-tomography image registration method based on digitally reconstructed radiographs”, Med. Phys. 21, 1749, 1994. 
[3] L. M. G. Brown and T. E. Boult. "Registration of planar film radiographs with computed tomography". Proc. IEEE MMBIA, 1996.

[4] Z"ollei L, “2D-3D rigid-body registration of x-ray fluoroscopy and CT images", MSc Thesis Dept. of Electrical Engineering and Computer Science, Massachusetts Institute of Technology, 2001.

[5] Russakoff, D.B.; Rohlfing, T.; Maurer, C.R., Jr.; "Fast intensity-based 2D-3D image registration of clinical data using light fields"; Computer Vision, 2003.

[6] Birkfellner W, Wirth J, Burgstaller W, et al., "A faster method for 3D/2D medical image registration---A simulation study", Phys Med Biol; 48:2665--2679, 2003.

[7] G. P. Penney, J. Weese, J. A. Little, P. Desmedt, D. L. G. Hill, and D. J. Hawkes, "A comparison of similarity measures for use in 2D-3D medical image registration," IEEE Trans. Med. Imaging, vol. 17, pp. 586-595, 1998.

[8] Daniel B. Russakoff, Torsten Rohlfing, Anthony Ho, Daniel H. Kim, Ramin Shahidi, John R. Adler Jr., Calvin R. Maurer Jr.: Evaluation of Intensity-Based 2D-3D Spine Image Registration Using Clinical Gold-Standard Data. WBIR 2003: 151-160, 2003.

[9] P. Viola and W. M. Wells III, Alignment by maximization of mutual information, (1995), pp. 16-23. IEEE, 1995.

[10] A. Collignon, F. Maes, D. Delaere, D. Vandermeulen, P. Suetens and G. Marchal, "Automated multimodality image registration based on information theory", Information Processing in Medical Imaging (Y. Bizais, C. Barillot and R. Di Paola, eds.), Kluwer Academic Publishers, Dordrecht, pp. 263-274, 1995.

[11] Matthew Mellor and Michael Brady, Phase Mutual Information as a Similarity Measure for Registration, Medical Image Analysis, 9(4), 330-343, 2005

[12] N. G. Kingsbury, Image processing with complex wavelets, Phil. Trans. Royal Society London A, Sept. 1999.

[13] Owens, R., Venkatesh, S., "On the classification of image features", Pattern Recognition Letters 11 (5), 339-349, 1990.

[14] Magnus Hemmendorff, Mats T. Andersson, Torbjorn Kronander, Hans Knutsson, "Phase-Based Multidimensional Volume Registration”, IEEE Trans. Med. Imaging 21(11): 1536-1543, 2002. 